\title{
An Informatics Framework to Support Surveillance System Interoperability in Minnesota
}

\author{
Samuel T. Patnoe ${ }^{\star 1,2}$, Martin LaVenture', Rebecca E. Johnson', Jennifer Fritz' \\ Barbara Frohnert', Geoffrey Mbinda', Karen Soderberg' ${ }^{1}$ and Bree Allen'
}

'Office of Health Information Technology, Minnesota Department of Health, St. Paul, MN, USA; ${ }^{2}$ Council of State and Territorial Epidemiologists, Atlanta, GA, USA

\section{Objective}

To create an informatics framework and provide guidance to help Minnesota's public health surveillance systems achieve interoperability and transition to standards-based electronic information exchange with external health care providers using the state's birth defects registry as an initial pilot program.

\section{Introduction}

The Minnesota Department of Health (MDH) needs to be able to collect, use, and share clinical, individual-level health data electronically in secure and standardized ways in order to optimize surveillance capabilities, support public health goals, and ensure proper follow-up and action to public health threats. MDH programs, public health departments, and health care providers across the state are facing increasing demands to receive and submit electronic health data through approaches that are secure, coordinated, and efficient; use appropriate data standards; meet state and federal privacy laws; and align with best practices. This framework builds upon existing informatics models and two past studies assessing health information exchange (HIE) conducted by the MDH Office of Health Information Technology (OHIT) to provide MDH surveillance systems with an outline of the key elements and considerations for transitioning to more secure, standards-based, electronic data exchange.

\section{Methods}

Development of the informatics framework incorporates information gathered in several phases. The first phase involves additional analysis of data collected from the MDH Informatics Assessment of Interoperability and $\mathrm{HIE}^{1}$ that was conducted in 2015 to evaluate the current state of interoperability and HIE readiness across the agency. The second phase involves a comprehensive environmental scan and literature review of existing standards, practices, models, toolkits, and other resources related to electronic HIE and interoperability. The third phase involves gathering additional information on programmatic needs, workflows, and capabilities through key informant interviews. Key informants include program managers, staff, and content-area experts from select MDH programs, the state's central information technology organization (MN.IT), and external health care provider organizations including hospitals.

Minnesota's birth defects registry, the Birth Defects Information System (BDIS), was selected as the pilot program because it was identified in the $2015 \mathrm{MDH}$ Informatics Assessment as having a high level of interest in implementing an interoperable and standardsdriven approach to electronic health data exchange. The BDIS is also exploring options for being designated as an eligible public health registry for Meaningful Use. As a pilot program for this project, the BDIS assists in the development and implementation of the informatics framework.

\section{Results}

The 2015 MDH Informatics Assessment identified and evaluated $21 \mathrm{MDH}$ programs with information systems that accept and manage clinical, individual-level health information. Among these $21 \mathrm{MDH}$ programs, wide variations exist regarding information system size (range, 400 to $10,000,000$ individuals), staffing numbers (range, 0.2 to 21 FTEs), budgets (range, $\$ 20,000$ to $\$ 1,876,000$ ), and other key characteristics. Despite these variations, programs identified similar barriers and needs related to achieving interoperability and electronic HIE. Areas of need include management and information technology support to make interoperability a priority; policies and governance; additional application functionality to support HIE; and additional skills for the workforce. Results from the environmental scan and key informant interviews will be incorporated with additional analyses of the $2015 \mathrm{MDH}$ Informatics Assessment to inform the development of an agency-wide informatics framework to support MDH programs in achieving interoperability.

\section{Conclusions}

MDH surveillance systems are calling for practical guidance to help implement and maintain a more efficient and effective way to electronically collect, use, and share health data with external and internal stakeholders. This informatics framework provides an outline of the key elements and considerations for achieving greater interoperability across MDH surveillance systems. Additional research is required to assess how system interoperability and HIE can improve data quality and advance population health goals.

\section{Keywords}

informatics; interoperability; HIE

\section{Acknowledgments}

This work is supported in part by the MDH and an appointment to the Health Systems Integration Program Fellowship administered by the Council of State and Territorial Epidemiologists (CSTE) and funded by the Centers for Disease Control and Prevention (CDC) Cooperative Agreement 3U38-OT000143-01S4.

\section{References}

1. Please contact mn.ehealth@state.mn.us for a copy of the $2015 \mathrm{MDH}$ Informatics Assessment.

\section{*Samuel T. Patnoe}

E-mail: sam.patnoe@state.mn.us 